\title{
ADVANCEMENTS IN TECHNOLOGY AND DESIGN OF BIOMIMETIC FLOW-SENSOR ARRAYS
}

\author{
C.M. Bruinink, R.K. Jaganatharaja, M.J. de Boer, E. Berenschot, M.L. Kolster, T.S.J. Lammerink, \\ R.J. Wiegerink, and G.J.M. Krijnen \\ Transducers Science and Technology, MESA ${ }^{+}$and IMPACT Research Institutes, University of \\ Twente, P.O. Box 217, NL-7500 AE Enschede (The Netherlands)
}

\begin{abstract}
This paper reports on recent developments to increase the performance of biomimetic flow-sensor arrays by means of several technological advancements in the fabrication procedures and corresponding sensor design optimizations. Advancements include fabrication procedures with higher process latitude and geometrical modifications of several parts of the flow sensor. The conclusive measurements in this paper support our sensormodel predictions for a 100-fold increase in acoustic sensitivity (down to oscillating flow amplitudes in the order of $1 \mathrm{~mm} \cdot \mathrm{s}^{-1}$ ) translating to substantially higher capacitive outputs in comparison to our first-generation biomimetic flow-sensor arrays.
\end{abstract}

\section{INTRODUCTION}

Crickets can extract aerodynamic information from many filiform flow-sensitive hairs (of various length, diameter and directivity) on two conical appendages (cerci) at the rear of their abdomen (Figure 1).

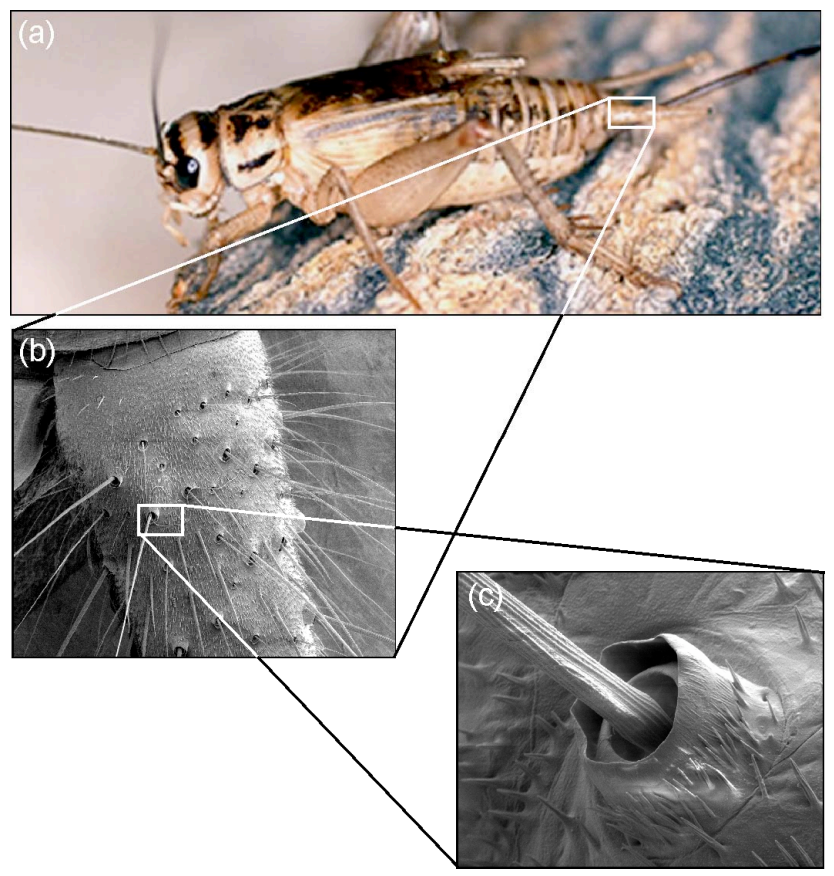

Figure 1: (a) Photograph of the Acheta Domesticus and (b,c) SEM images of the base part of a cercus and a closeup of a filiform flow-sensitive hair (courtesy of J. Casas, IRBI, University of Tours).

The longer filiform hairs (500-1000 $\mu \mathrm{m})$ are highly perceptive to low-frequency sound waves [1] and can sense air velocities as small as $30 \mu \mathrm{m} \cdot \mathrm{s}^{-1}$ [2], enabling crickets to identify and escape from predators.

Several research groups have been working on modeling, fabrication and optimization of biomimetic flow-sensing devices consisting of arrays of many flowsensors. MEMS fabrication technology is very practical in the realization of these devices for its capabilities of parallel fabrication and integration of flow sensors into large sensor arrays. The additional value of this type of arrays is that the flow-sensitive hairs allow measurement of flows with high spatial resolution and therefore could facilitate complex flow pattern measurements. Several types of biomimetic flow-sensors can be found in literature incorporating various transduction mechanisms. These include piezoresistive sensing [3], capacitive sensing [4] and switching [5].

This paper describes the successful continuation of the fabrication and design of flow-sensor arrays with capacitive sensing [4] for reasons of high intrinsic sensitivity in combination with low power dissipation and promising performance.

\section{SENSOR-MODEL DESIGN}

In our sensors, capacitive transduction relies on the tilting of a membrane by drag forces acting on the receptive hair of the sensor (Figure 2). The metallic electrodes on top of the membrane form capacitors with the underlying common electrode. A tilt at the rotational axis of the membrane by the deflection of the drag-force receptive hair causes a differential change in capacitance and therefore provides a means of measuring air flows.

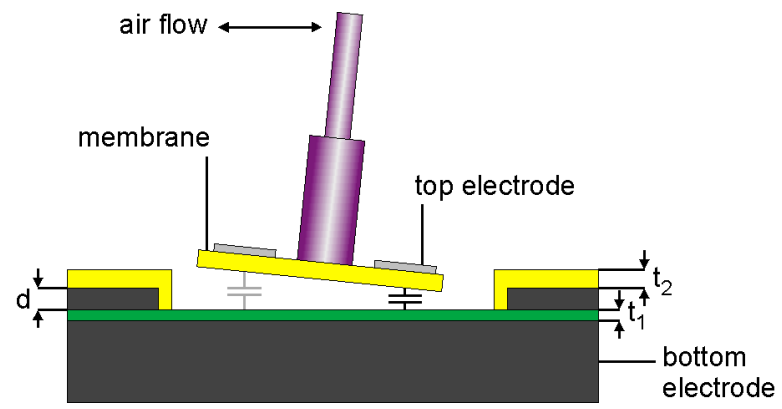

Figure 2: Schematic representation of the operational principle of capacitive sensing.

Application of the aerodynamic models for the filiform hairs of crickets [1] to our biomimetic flow sensors results in several essential design considerations for optimal sensor performance [6]. For comparison of the performance of the biomimetic flow sensors with the filiform hairs of crickets, we define a figure of merit (FoM) [6] as the product of usable bandwidth and the low-frequency sensitivity:

$$
\text { FoM }=\text { bandwidth } \cdot \text { sensitivity } \equiv \omega_{0} \cdot \frac{T_{d}}{S}
$$

(S: torsional spring constant) 
The bandwidth of the flow sensors is proportional to the mechanical resonance frequency given by:

$$
\sqrt{\frac{S}{I}} \propto \sqrt{\frac{S}{\rho \cdot L^{3} \cdot D^{2}}}
$$

(L: hair-length; D: hair-diameter; $\rho$ : density of the hair; I: moment of inertia)

For hair lengths above the boundary layer thickness (e.g. at a frequency of $100 \mathrm{~Hz}$, this translates to a minimum hair length of $440 \mu \mathrm{m})$ and for the frequency $(10-$ $1000 \mathrm{~Hz})$, hair-length $(100-1000 \mu \mathrm{m})$ and hair-diameter $(5-50 \mu \mathrm{m})$ ranges of interest, the sensitivity of the flow sensors is roughly proportional to [6]:

$$
\frac{L^{2} \cdot D^{1 / 3}}{S}
$$

By combining equations 2 and 3, the FoM becomes proportional to:

$$
F O M \propto \sqrt{\frac{L}{\rho \cdot S \cdot D^{4 / 3}}}
$$

Equation 4 provides clear directions for (design) modifications to enhance the overall performance of the first-generation flow-sensor arrays (see Figure 3a) [4].
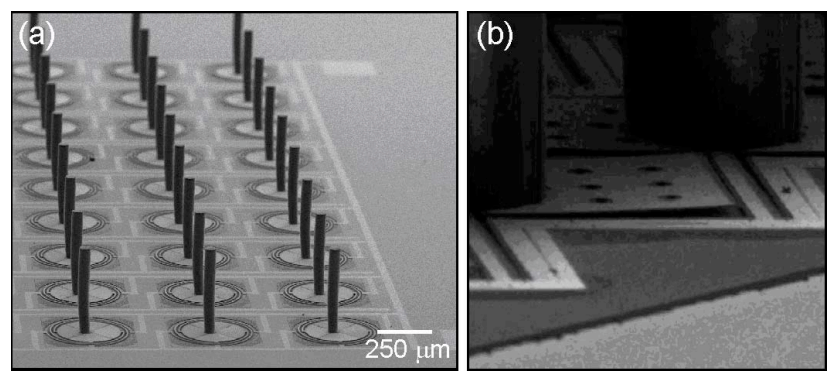

Figure 3: (a) SEM image of the first-generation flowsensors arrays with $450 \mu \mathrm{m}$-long hairs and spiral suspensions. (b) SEM image of the membrane curvature $(\delta \approx 2.5 \mu \mathrm{m})$ as a result of the high tensile stress in the chromium electrodes on top of the membrane.

These modifications include the incorporation of a twostep photolithographic procedure for the fabrication of longer SU-8 hairs (to increase drag-torque pick-up), smaller inter-electrode gaps (to increase the capacitive sensitivity) and adjustments in the lateral dimensions of the torsion beams (to reduce torsional stiffness). Additionally, experimental findings illustrate the need of low-stress electrode materials in combination with a more optimal electrode design to counteract for the membrane curvature (Figure $3 b$ ). The effect of the curvature $(\delta)$ on the sensitivity $\eta$ is given by [6]:

$$
\begin{aligned}
& \eta=\frac{w \cdot l^{2}}{\left(d_{0}+\delta\right) \cdot d_{0}} \\
& \text { with } d_{0}=d \cdot \frac{t_{1}+t_{2}}{e_{r}} \text { (see Figure 2) }
\end{aligned}
$$

( $\delta$ : curvature at the rim of the membrane; $d_{o}$ : dielectric thickness (Figure 2); w: membrane-width; l: membranelength; $\mathcal{E}_{r}$ : dielectric constant of silicon nitride)
The effects of these modifications on the sensitivity are shown as (theoretical) sensitivity improvement factors in Table 1.

Table 1: Technological sensitivity improvement factors for various design parameters.

\begin{tabular}{lcc}
\hline Design parameter & & Factor \\
\hline & & \\
inter-electrode gap & $1.0 \mu \mathrm{m} \rightarrow 0.6 \mu \mathrm{m}$ & 2.7 \\
hair length & $450 \mu \mathrm{m} \rightarrow 900 \mu \mathrm{m}$ & 4.0 \\
hair diameter & $50 \mu \mathrm{m} \rightarrow 25 \mu \mathrm{m}$ & 0.8 \\
membrane curvature & $2.5 \mu \mathrm{m} \rightarrow$ no bending & 4.3 \\
membrane shape & circular $\rightarrow$ rectangular & 1.5 \\
torsion beams & & 1.8 \\
$\quad$ - length & $75 \mu \mathrm{m} \rightarrow 100 \mu \mathrm{m}$ & \\
$\quad$ - width & $10 \mu \mathrm{m} \rightarrow 5 \mu \mathrm{m}$ & \\
\hline
\end{tabular}

The adjustment of the hair-diameter (Table 1) comes at the penalty of a slight reduction in drag-force, however, the smaller diameter considerably decreases the moment of inertia (by about 65\%), allowing smaller torsional stiffness while still maintaining sufficient bandwidth.

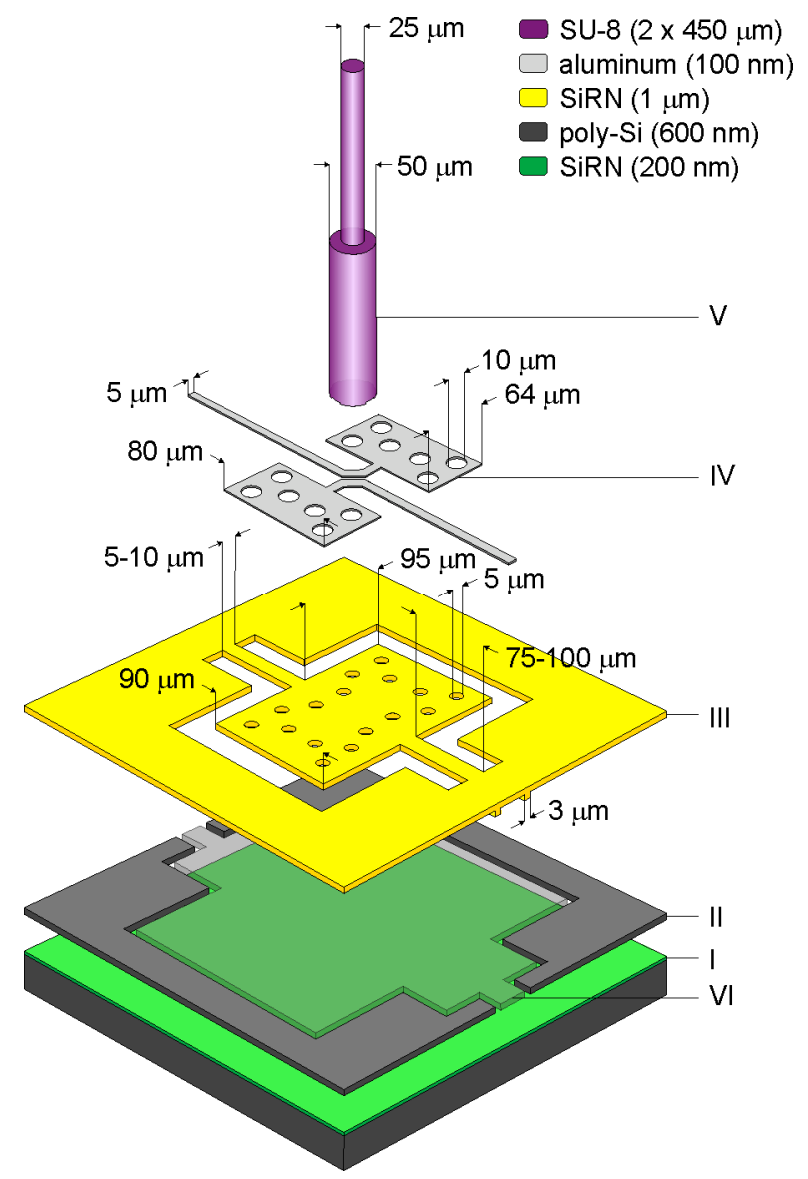

Figure 4: Schematic representation of the fabrication of the artificial hair sensor, including the dimensions.

(I) LPCVD of the protective SiRN layer; (II) LPCVD of the sacrificial poly-Si layer and patterning of the protection trenches; (III) LPCVD of the SiRN layer and patterning of the membrane/torsion beam structures; (IV) sputtering of aluminum and patterning of the top electrodes; (V) two-step $S U-8$ processing of the hair; (VI) sacrificial layer etching. 


\section{FABRICATION}

A thin silicon-rich nitride (SiRN, $200 \mathrm{~nm}$ ) layer was deposited on a highly conductive silicon wafer (as the common bottom electrode) by low-pressure chemical vapor deposition (LPCVD, Figure 4-I) for protection of the wafer during later sacrificial layer etching. The sacrificial poly-silicon (poly-Si) layer was deposited to a thickness of $600 \mathrm{~nm}$ by LPCVD and patterned by reactive-ion etching (RIE) to form protection trenches (Figure 4-II). A second $1 \mu \mathrm{m}$-thick SiRN layer was deposited by LPCVD to form the actual membrane and torsion beams after etching by RIE (Figure 4-III) and to complete the protection of specific areas in the poly-Si layer. From past experiments it is known that the deposition and design of the electrode systems is of prime importance for the sensor performance (due to membrane curvature by the internal stress in the metal layer). After performing several test runs for stress and resistance measurements of the electrode systems, the thickness of the aluminum layer was set to $100 \mathrm{~nm}$ resulting in lowstress electrode systems. After sputtering the low-stress aluminum by low-power sputtering at room temperature, the electrode systems were patterned by wet-etching in standard resist developer (Figure 4-IV). Use of the developer has several advantages over the conventional aluminum etchants, (low etch rate, room temperature etching) and results in the definition of the electrode systems with high fidelity $(<1 \%$ deviation with lateral dimensions of the resist mask). For the fabrication of 900 $\mu \mathrm{m}$-long SU-8 hairs, processing was done by a sequential exposure procedure of two $450 \mu \mathrm{m}$-thick SU-8 layers imposed by the maximum exposure thickness of only $700 \mu \mathrm{m}$ due to UV light adsorption.

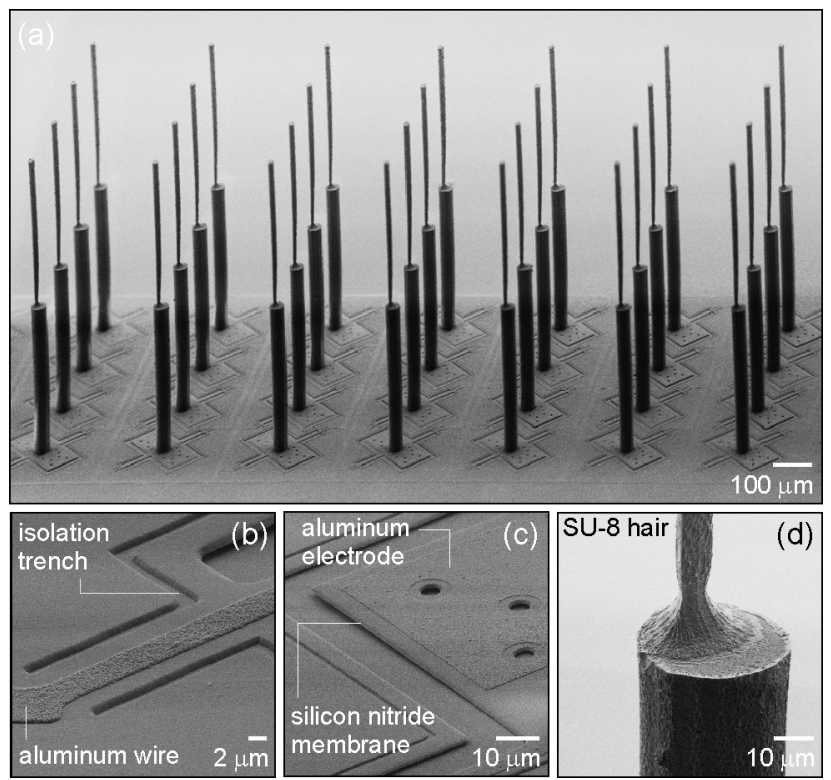

Figure 5: Series of SEM images illustrating in detail the final second-generation flow-sensor array: (a) part of the array with $900 \mu \mathrm{m}$-long hairs, (b) part of the aluminum wiring, (c) close-to-flat membrane with aluminum electrode, and (d) connection between two segments of the SU-8 hair. Note: charging during SEM imaging is causing parts of the membrane to stick to the substrate (image c).

Additionally, this two-step photolithographic procedure enables the fabrication of hairs consisting of two segments of different diameter (Figure 4-V). Finally, the sacrificial layer etching of the poly-silicon for releasing the sensor from the substrate (Figure 4-VI) was done on the frontside using a glass cover over the entire wafer to protect the sensor parts (membrane, electrodes and SU-8 hair) from ion-bombardment during etching.

The series of SEM images in Figure 5 illustrate in detail the resulting second-generation flow-sensor array, consisting of 124 flow-sensors in a parallel configuration with $45^{\circ}$ rotation with respect to the longitudinal axis of the sensor array. The significant reduction of the membrane curvature, from $2.5 \mu \mathrm{m}$ (in case of chromium in the first-generation sensors) to about $100 \mathrm{~nm}$ (light interference microscopy data not shown), by using lowstress aluminum as the electrode material is seen in Figure 5c.

Overall, the present fabrication modifications result in second-generation flow sensors with close-to-flat membranes, longer hairs and a smaller inter-electrode gap.

\section{ACOUSTIC MEASUREMENTS}

Acoustic characterization of this flow-sensor array was carried out with a directional flow-sensitivity measurement set-up (Figure 6) using oscillating flows with amplitudes in the range of $1-100 \mathrm{~mm} \cdot \mathrm{s}^{-1}$ and measuring the output at different source angles with respect to the rotational axis of the flow sensor.

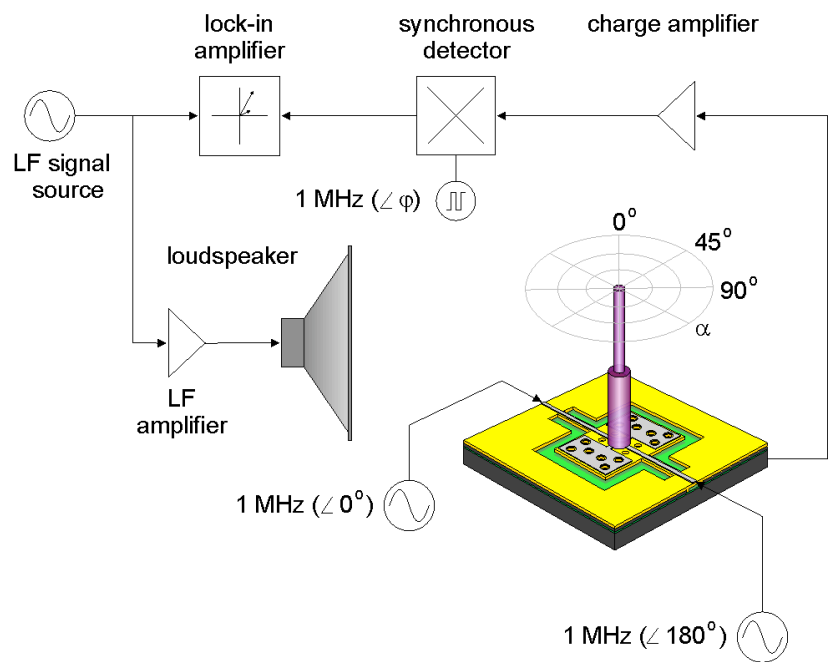

Figure 6: Schematic representation of our acoustic flow measurement set-up, illustrating the essential components for performing acoustic measurements.

This measurement set-up allows the characterization of the flow-sensor arrays with near field acoustic flows from a loudspeaker by employing capacitive amplitude modulation of two $1 \mathrm{MHz}$ electrical signals [7]. The electrical signals are taken $180^{\circ}$ out of phase for acquiring a differential-mode rotational signal of the flow sensor. After the charge amplifier and synchronous detector (by a multiplier circuit), the resulting base-band signal is fed to a lock-in amplifier to obtain the LF signal. Figure 7 shows the results of such a differential-mode rotational measurement on our present flow-sensor arrays. The signal follows to a large extend a figure-of-eight, indicating the preferential sensitivity of the flow sensor in the direction perpendicular to the rotation axis. 


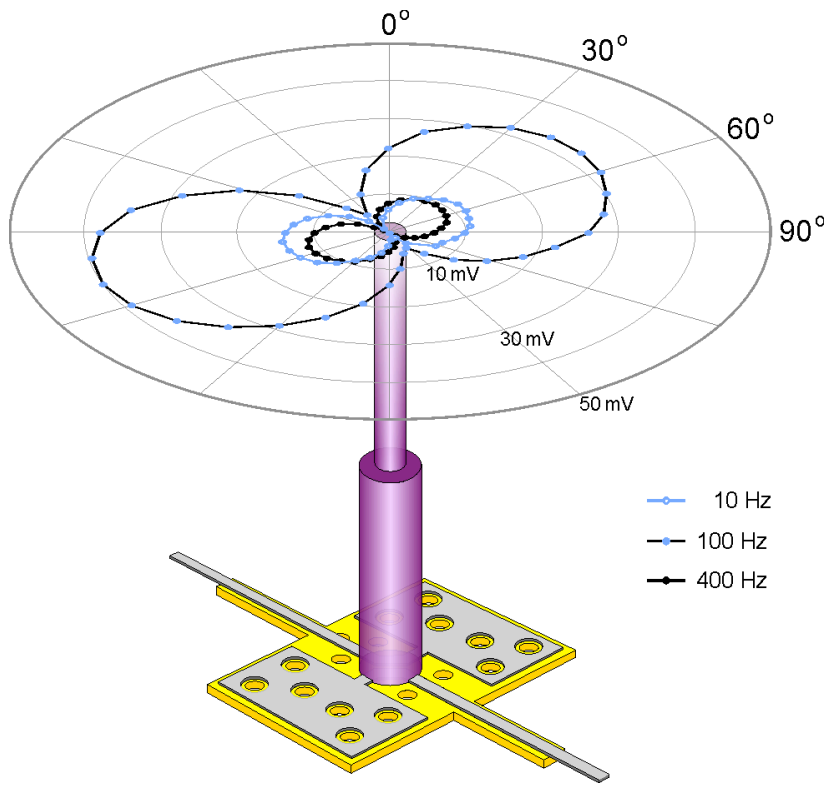

Figure 7: Superposition of the acoustic measurements at three different frequencies on the flow-sensor.

Sensitivity measurements were taken by using the same measurement set-up, including appropriate filtering and noise amplitude measurements (Figure 8). The lower flow detection limit is given by the intersection of the ( $1 \mathrm{~Hz}$ bandwidth) measured signals with the larger bandwidth noise values. Depending on signal frequency and operating bandwidth, minimum flow amplitudes of about $2 \mathrm{~mm} \cdot \mathrm{s}^{-1}$ can be obtained. This measurement supports the (theoretical) 100-fold increase in sensitivity (see Table 1) due to the present modifications in the second-generation flow-sensor arrays.

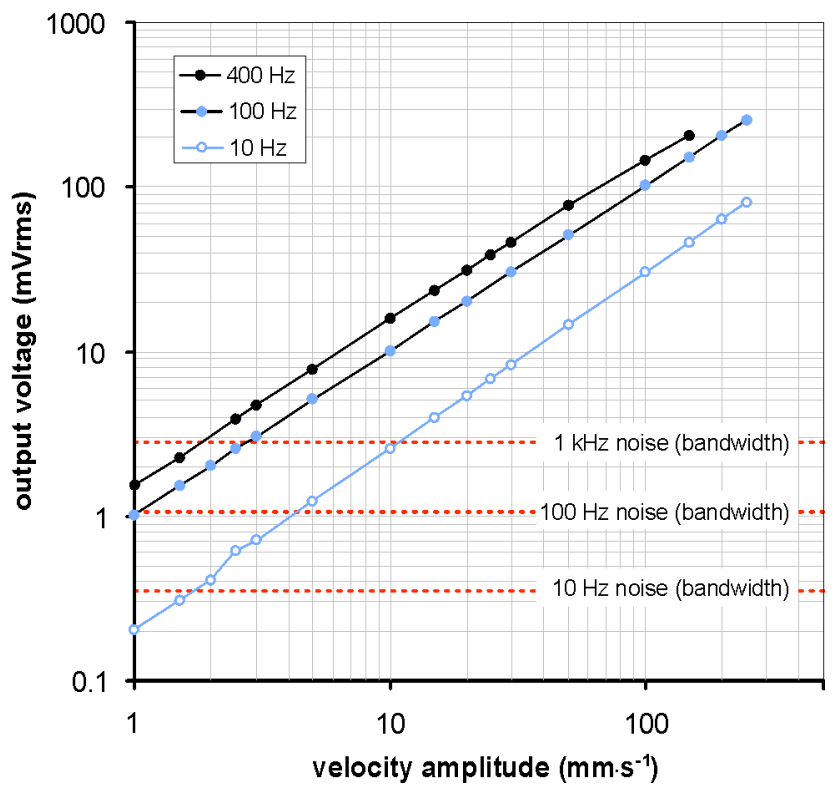

Figure 8: Sensitivity measurements at three different frequencies (dots), with the dashed red lines indicating the RMS noise level for three bandwidths.

Significant improvements in the noise levels are foreseen by further optimization of the electronics.

\section{CONCLUSIONS}

We have shown significant advancements in sensor technology and design that enable us to fabricate biomimetic flow-sensor arrays with sensitivities on the order of $1 \mathrm{~mm} \cdot \mathrm{s}^{-1}$. Future research will concentrate on further enhancements by incorporation of different torsion beam constructions (for lowering the torsional spring stiffness), searching for optional materials to decrease the density of the hairs, and by the definition of separate bottom electrodes (for reduction of parasitic capacitances) in order to match the performance of our biomimetic flowsensor arrays to the sensory hairs of crickets with sensitivities down to $30 \mu \mathrm{m} \cdot \mathrm{s}^{-1}$.

\section{REFERENCES}

[1] (a) J.A.C. Humphrey, R. Devarakonda, I. Iglesias, F.G. Barth, "Dynamics of arthropod filiform hairs. I. Mathematical modeling of the hair and air motions" Phil. Trans. R. Soc. Lond. B, 340, pp. 423-444, 1993. (b) T. Shimozawa, T. Kumugai, Y. Baba, "Structural scaling and functional design of the cercal windreceptor hair of crickets", J. Comp. Physiol. A, 183, pp. 171-186, 1998.

[2] T. Shimozawa, J. Murakami, T. Kumagai, Sensors and Sensing in Biology and Engineering (Chapter 10), ed. F.G. Barth, J.A.C. Humphrey and T.W. Secomb, Springer, Vienna, 2003.

[3] Y. Ozaki, T. Ohyama, T. Yasuda, I. Shimoyama, "Air flow sensor modeled on wind receptor hairs of insects", in Proc. IEEE Int. Conf. MEMS, Miyazaki, 2000, pp. 531-536.

[4] J.J. van Baar, M. Dijkstra, R. Wiegerink, T.S.J. Lammerink, R. de Boer, G.J.M. Krijnen, "Arrays of cricket-inspired sensory hairs with capacitive motion detection", in Proc. IEEE Int. Conf. MEMS, Miami, 2005, pp. 646-649.

[5] W.J. Kuipers, J.J. van Baar, M. Dijkstra, R.J. Wiegerink, T.S.J. Lammerink, J.H. de Boer, G. J.M. Krijnen, "Drag force actuated bistable microswitches for flow sensing", in Proc. IEEE Int. Conf. MEMS, 2006, pp. 658-661.

[6] G.J.M. Krijnen, A. Floris, M. Dijkstra, T.S.J. Lammerink, R. Wiegerink, "Biomimetic micromechanical adaptive flow sensor arrays", in SPIE Microtechnologies for the New Millennium, Gran Canaria, 2007, pp. 6592-6616.

[7] M. Dijkstra, J.J. van Baar, R.J. Wiegerink, T.S.J. Lammerink, J.H. de Boer, G.J.M. Krijnen, "Artificial sensory hairs based on the flow sensitive receptor hairs of crickets", J. Micromech. Microeng., 15, pp. S132-S138, 2005.

\section{ACKNOWLEDGEMENTS}

The authors want to thank Mark Smithers for highresolution SEM imaging, our colleagues in the EU project CILIA (www.cilia-bionics.org) for stimulating discussions and input to this work, the EU for financial support by the Future and Emergent Technologies arm of the IST Programme in the $6^{\text {th }}$ Framework Programme and NWO/STW for financial support in the framework of VICI project BioEARS. 